\title{
Résultats obtenus par l'emploi de la photographie sous-marine sur les fonds du large de Roscoff
}

\author{
Louis CABIOCH \\ Station Biologique de Roscoff, Roscoff, France
}

\begin{abstract}
Results obtained by employing underwater photography on the sea-bottom off Roscoff. An underwater camera has been used for the study of the sea-bottom of the Channel, near Roscoff. Together with observations on the dimensions of the boulders, which are scattered over the bottom, the analysis of the photographs shows that the main features of the benthic populations and their distribution have not changed since 1959, when they were studied by systematic dredgings. Examples of uniformity or local variability of the populations are given, and the sharpness of the boundaries of the agglomerations of Ophiothrix fragilis is shown. Some features of the behaviour of the benthic animals have been observed: Ophiothrix fragilis assume a "feeding posture", with their arms upswept, when the speed of the tidal stream is lower than 0.8 knots; the downfall of the food particles is then easier. O. fragilis creep on the substratum when the water current exceeds 0.8 knots.
\end{abstract}

\section{INTRODUCTION}

La Station Biologique de Roscoff a entrepris, depuis quelques années, sous la direction de Monsieur le Professeur Teissier, une étude systématique des fonds du large de notre région. Les techniques classiques de dragage et de sondage d'abord utilisées ont permis d'étudier la géologie et les peuplements benthiques de la Manche occidentale (Boillot 1960, 1964; CaBioch 1961, 1965). Les imperfections des méthodes de dragage sont bien connues; la drague fonctionne diversement selon la nature du fond et ne fournit aucun renseignement quantitatif précis. Elle est traînée sur un parcours plus ou moins long et le mélange qui en résulte rend difficile l'observation du passage d'un peuplement à un autre. La nature particulière des fonds de notre région, constitués essentiellement de sédiments grossiers, de cailloutis et de blocs de toutes tailles, s'oppose, en outre, à l'utilisation généralisée des engins de récolte quantitative.

L'étude plus précise des riches épibioses du large de Roscoff nécessitait donc l'emploi de méthodes d'observation directe. L'acquisition, par le laboratoire, d'un appareil de prises de vues sous-marine automatique a permis d'aborder cette étude. Les dimensions et les moyens dont dispose notre navire de recherche, le Pluteus II, ainsi que l'importance des courants de marée et la nature du substrat en Manche, imposaient certaines conditions. Il fallait un appareil peu encombrant, aisément maniable, disposé verticalement et comportant une source autonome d'énergie électrique. L'appareil 
Thorndyke, construit par la société «Alpine Geophysical Associates», présentait toutes ces caractéristiques et s'est révélé d'un emploi relativement aisé à bord d'un navire de faible tonnage comme le «Pluteus II».

\section{METHODES D'UTILISATION}

La mise à l'eau de l'appareil s'effectue à l'aide d'un cable que l'on engage dans une poulie compteuse afin de connaître à chaque instant la profondeur d'immersion. On suit, en outre, la descente de l'engin et son contact avec le fond à l'aide du sondeur où s'enregistrent les échos renvoyés par les deut boitiers de l'appareil (appareil de prises de vues et flash). Un contrepoids provoque, à son arrivée sur le fond, le déclenchement de la prise de vues. L'axe optique de la camera est incliné de 36 degrés par rapport à la verticale et la vue se trouve prise à une distance approximative de 2,50 mètres $\mathrm{du}$ fond. L'appareil est remonté immédiatement après et le déroulement automatique du film rend nécessaire une attente de 1 minute 40 secondes avant la prise de vue suivante.

Deux modes d'utilisation ont été expérimentés. Une première méthode permet d'établir ou de préciser les cartes générales de répartition des différents types de fonds. Dans ce cas, on photographic point par point une région déterminée du domaine marin suivant un quadrillage régulier à mailles assez larges. L'appareil est remonté sur le pont entre les stations et l'expérience montre que, pour des intervalles d'un demi mille et par 60 à 100 mètres de profondeur, les stations peuvent être effectuées à la fréquence de sept ou huit par heure.

Une seconde méthode permet d'étudier l'uniformité ou la variabilité locale des peuplements. On prend, à partir d'un point donné, une série de vues très proches les unes des autres. Remonté entre deux eaux à quelques mètres au-dessus du fond après chaque prise de vue, l'appareil est redescendu au bout de deux minutes. Le bateau dérive sous l'effet du courant et du vent et les photographies se trouvent réparties en une série linéaire serrée dans le sens du déplacement. Les courants de marée sont, à Roscoff, la cause principale de cette dérive et la distance minimum entre deux vues successives en dépend essentiellement. Le choix de l'heure de l'opération par rapport à celle de l'étale de courant offre donc, à cet égard, diverses possibilités.

Il convient, cependant, de remarquer que l'intensité de ces courants en période de vive-eau ( 3 à 5 noeuds au maximum au Nord de l'île de Batz) limite considérablement les possibilités d'utilisation de l'appareil, du fait de l'accroissement de la turbidité de l'eau. Les fortes houles ont de semblables effets à faible profondeur. Craig \& PrIEsTLEY (1964) ont également rencontré de telles difficultés.

\section{PREMIERS RESULTATS}

Trois cents stations ont été photographiées au cours de l'année 1965, en un premier temps, sur les fonds de l'Ouest, du Nord et du Nord-Est de l'île de Batz, pour 
lesquels une carte des peuplements avait été établie par dragage lors d'une étude précédente (САBıOCH 1961). Il est alors possible de comparer, à plusieurs années d'intervalle, les résultats obtenus par les deux méthodes. Une expérience de télévision sous-marine a, d'autre part, été entreprise parallèlement à la campagne d'exploration photographique.

Le dépouillement détaillé des documents obtenus n'est pas l'objet de la présente note. Nous indiquerons simplement les principaux points pour lesquels la photographie sous-marine a permis d'apporter un complément d'information.

\section{Nature du substrat}

Les fonds situés au Nord de la région de Roscoff, au delà de la limite des 65 mètres, sont constitués par un vaste plateau en pente douce, composé de calcaire éocène recouvert d'une nappe de cailloutis et de graviers résultant de la dissociation d'un conglomérat quaternaire (BolLlot 1964) et dont la drague ne récolte que les plus petits éléments. L'exploration photographique et l'emploi de la télévision sousmarine ont révélé que certains de ces blocs peuvent atteindre, en réalité, une envergure de 1 à 2 mètres. Ces blocs isolés apparaissent, sur certains fonds (fonds à Ophiures du Nord-Est de Batz), posés sur une nappe uniforme de cailloutis de faible dimension. Boillot interprète leur présence comme le résultat d'un délestage des glaces flottantes qui serait intervenu au moment du réchauffement climatique ayant succédé à la dernière glaciation (BouHot et al. 1965). Beaucoup plus abondants en d'autres points (Tachad, Trou Santic), ils forment de véritables chaos de blocs émoussés (Fig. 1a).

\section{Permanence des grandes unités de peuplement}

Deux épibioses principales peuplent les fonds de cailloutis situés entre la ligne des 65 mètres et une ligne située à 7 ou 8 milles de distance de la côte, dans la région de Roscoff. Ce sont les fonds à Ophiotbrix fragilis et les fonds à Axinella dissimilis.

Il était intéressant de rechercher, par exemple, dans quelle mesure les vastes populations d'Ophiures cartographiées en 1959 (САBIOCH 1961) occupaient encore les mêmes positions. Le quadrillage photographique, tout en révélant leur grande densité (200 à 300 individus par mètre carré), a montré que leurs limites étaient demeurées sensiblement les mêmes que celles qui avaient été observées six ans auparavant lors des dragages systématiques. Cette permanence est particulièrement nette dans le cas du vaste fond d'Ophiures du Nord-Est de Batz connu sous le nom de «Trou aux Singes». Ce fond occupe une superficie de 25 kilomètres carrés, et les photographies effectuées aux mêmes points que les dragages y révèlent le même peuplement. Il fait place, vers l'Ouest, aux fonds à Axinella dissimilis du Tachad dont les limites semblent également n'avoir pas varié. La bordure nord de la plupart des fonds à Ophiures moins nette, a, par contre, subi quelques légères fluctuations. 


\section{Uniformité ou variabilité locale des peuplements}

La méthode des prises de vues en série permet d'apprécier l'uniformité plus ou moins grande des différents types de fonds. Un exemple peut en être donné par une série de vingt cinq vues prises dans les limites du fond à Ophiures du «Trou aux Singes», à une distance de 60 mètres environ les unes des autres. Tous les clichés montrent une population d'Ophiures extrêmement dense et relativement uniforme, qui s'appauvrit seulement, par places, lorsque un dépôt de sable.s'accumule à l'abri de blocs un peu plus gros. On observe, ça et là, la présence de quelques individus d'Ophiocomina nigra. Des colonies d' Alcyonium digitatum parsèment également le fond assez régulièrement. On conçoit aisément que, dans un tel type de fond, la longueur du trait de drague n'ait pas une importance considérable.

Il n'en est pas de même pour les fonds à Axinella dissimilis (fonds du «Tachad», situés à 80 mètres de profondeur) connus auparavant comme des fonds durs où la drague remonte, outre quelques cailloux parfois accompagnés de sable coquillier grossier, une faune essentiellement composée par des espèces sessiles arrachées à leur substrat. Cette faune comprend, principalement, de grandes Eponges (Axinella dissimilis, Axinella agnata, Phakellia ventilabrum), des Hydraires, des Bryozoaires (Hippodiplosia foliacea, Porella compressa, Cellaria) et l'ascidie coloniale Diazona violacea. L'observation des dragages laissait à penser que l'ensemble de cette faune devait être fixé sur des fonds de roche ou de gros blocs, légèrement accidentés. Rien ne permettait de soupçonner l'hétérogénéité que la photographie a révélée. Les clichés effectués en série dans cette région, à une distance de 40 mètres les uns des autres, ont montré, en effet, une alternance rapide entre des fonds réguliers, parsemés de cailloux de faible et moyenne dimensions, et des chaos de blocs émoussés, bien plus grands, de plus d'un mètre d'envergure parfois (Fig. 1a). Les fonds réguliers (Fig. 1b) sont recouverts par une riche épifaune où l'on reconnait en particulier les grandes Eponges caractéristiques: Axinella dissimilis et Phakellia ventilabrum. L'épifaune des chaos semble plus pauvre mais on y remarque la présence de colonies de Porella compressa fixées sur le sommet des blocs (Fig. 1a). La distribution de la faune n'est donc pas homogène dans l'ensemble des fonds décrits sous le nom de "Communauté à Axinella dissimilis et Phakellia ventrilabrum» (САBIOCH 1961).

\section{Mode de passage d'un type de peuplement à un autre}

Les limites entre les différents peuplements benthiques ont été déduites de l'observation d'un grand nombre de dragages. Cette méthode laisse, néanmoins subsister une certaine imprécision quant à la manière dont s'effectue le passage d'une unité de peuplement à une autre. Conjointement avec les observations effectuées à l'aide de la télévision, l'emploi de la photographie sous-marine nous apporte de notables précisions sur ce point. On peut ainsi étudier, par exemple, la région marginale des fonds à Ophiures. Des photographies ont été prises, dans ce but, à une distance de 40 mètres les unes des autres, sur la bordure occidentale du fond à Opbiothrix fragilis du "Trou aux Singes». Les clichés successifs montrent, sans variation appréciable de la nature du 

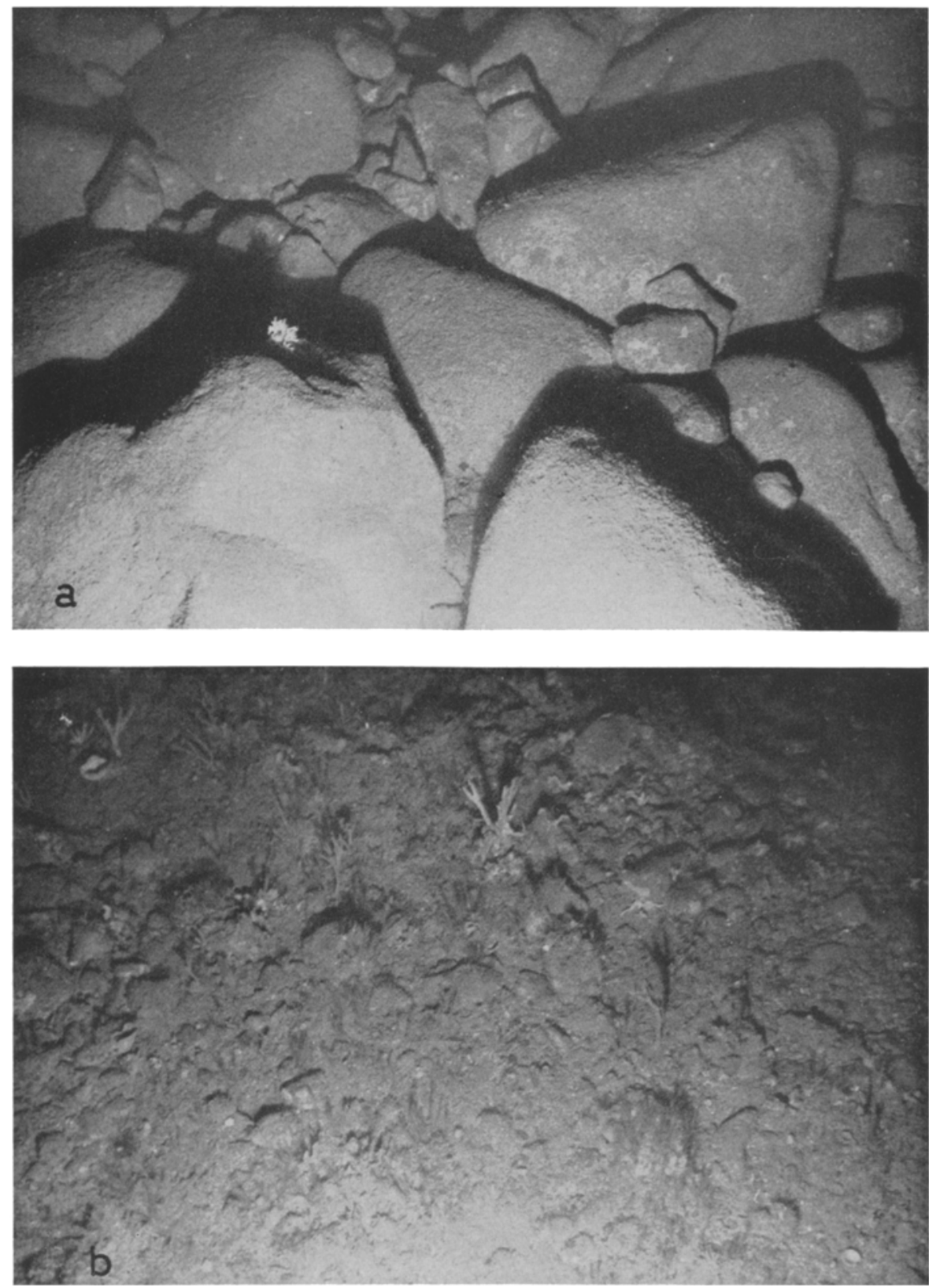

Fig. 1: Fonds à Axinella dissimilis du Tachad (N. de l'île de Batz), à 3 milles au N. du Pen ven, par $75 \mathrm{~m}$ de profondeur. (a): Chaos de blocs à Porella compressa. (b): Fond plus régulier à Axinella dissimilis (au milieu du champ vers le haut) et Phakellia ventilabrum (forme en entonnoir, en haut, à gauche). Les deux vues représentent des fonds situés à $40 \mathrm{~m}$ de distance environ l'un de l'autre. Largeur approximative du champ: $2 \mathrm{~m}$ 
substrat, soit la présence de populations denses d'Ophiotbrix, soit leur absence presque totale. Il semble donc que la disparition des agglomérations d'Ophiures s'effectue de façon très rapide en ce point. Une exploration continue des fonds situés à la bordure orientale de ce même banc, à l'aide de la télévision sous-marine, a permis d'observer l'apparition brusque de ce peuplement qui acquiert presque sans transition la densité qui sera la sienne sur tout le reste du trajet.

\section{Ethologie des animaux benthiques}

L'étude des documents photographiques permet également de mettre en évidence certains aspects du comportement des animaux benthiques. Les bancs d'Ophiures offrent, à nouveau, à cet égard, un terrain d'observation intéressant. Les soixante-douze clichés effectués dans ces fonds se répartissent, en effet, aisément en deux séries selon la position des animaux sur le fond. Ceux-ci, dans un premier cas, dressent vers le haut un nombre variable de leurs bras, tandis que la face orale repose au contact du substrat ou s'en décolle plus ou moins obliquement (Fig. 2a). Ces prairies ondulantes de bras dressés rappellent nettement les phénomènes observés en Méditerranée par Peres \& Picard (1958), Czihak (1959), Riedl (1961) et Guille (1965) dans le cas d'Opbiotbrix quinquemaculata.

D'autres vues montrent, au contraire, les Ophiures dans une position apparente de reptation. Les bras, souvent mêlés à ceux des individus voisins, sont alors posés sur le fond et ne se décollent que sporadiquement vers le haut (Fig. 2b). L'aspect de prairie ondulante disparait. Cette dernière disposition avait déjà été observée par Vevers (1952), Mac Intyre (1956), Blacker \& Woodhead (1965).

Certaines observations complémentaires, menées parallèlement aux prises de vues, ont mis en évidence une nette corrélation entre ces variations du comportement des populations d'Ophiures et les conditions hydrodynamiques. La vitesse du courant de surface a été mesurée lors des stations photographiques. Il suffit, pour cela, de calculer, à l'aide du système de navigation Decca, la dérive du bateau à l'arrêt pendant un temps déterminé, dans des conditions favorables de vent nul ou faible. Les résultats obtenus concordent d'ailleurs bien avec les données des cartes de courants de marée établies par le Service Hydrographique de la Marine et qui permettent de connaître la vitesse du courant dans le cas où la mesure directe n'a pu être effectuée.

Il apparait ainsi que la position dressée des bras des Ophiures correspond à de faibles courants (de vitesse inférieure à 0,8 noeud en surface). La position prostrée se manifeste, au contraire, lorsque le courant devient plus intense, sa vitesse étant alors supérieure à 0,8 noeud en surface.

Le mode de nutrition d'Ophiothrix fragilis fait intervenir plusieurs mécanismes différents: broutage, capture des aliments par mouvements des bras (VEvers 1956), dilacération d'animaux morts par les podia buccaux (NagabHushanam \& Colman 1959) ou bien encore récolte de particules par les sécrétions adhésives produites par les podia (v. UeXKüLl 1905, VeVERs 1956) et peut-être aussi par les épines (BuCHANAN 1963). Fontaine (1965) interprète la position dressée des bras d'Opbiotbrix fragilis comme une attitude de capture microphage; il résume, en outre, l'ensemble des données 



Fig. 2: Fonds à Ophiothrix fragilis du «Trou aux Singes». (a): 6 milles au N.-N.-W. des Duons, $75 \mathrm{~m}$. Ophiures en position dressée. Vitesse du courant de marée en surface: 0.3 noeud. (b): 4 milles au N. des Duons, $65 \mathrm{~m}$. Ophiures en position rampante. Vitesse du courant: 1,7 noend 
actuellement connues sur ce sujet. Cette position de capture correspond, à Roscoff, aux moments où les courants de marée sont les plus faibles. Corrélativement, la sédimentation des particules doit alors se produire plus aisément, favorisée, en outre, très vraisemblablement, par le freinage qu'exercent, dans les derniers centimètres, la multitude de bras dressés. Le rythme des courants de marée règle donc, dans une certaine mesure, l'activité nutritive d'Opbiotbrix fragilis.

Les fonds à Opbiotbrix sont également habités, en moins grande abondance, par des individus d'Ophiocomina nigra dont Fontaine (1965) décrit en détail la posture de capture microphage. Moins accusée que celle d'Ophiothrix fragilis, cette position apparait moins nettement sur les photographies. On l'observe cependant sur quelques vues, trop peu nombreuses pour que l'on puisse conclure, pour le moment, à un rythme comparable à celui d'Ophiothrix fragilis.

\section{RESUMÉ}

1. Un appareil de photographie sous-marine automatique a été utilisé pour l'étude des fonds de la Manche Occidentale, dans la région de Roscoff. Les méthodes d'utilisation sont décrites.

2. La connaissance de la nature du substrat a été améliorée: la photographie montre la présence, sur certains fonds, de blocs de grande dimension.

3. La permanence générale des grandes unités de peuplement sur les fonds du large de Roscoff apparait par comparaison entre les photographies et le résultat des dragages effectués aux mêmes points six ans auparavant.

4. L'étude de clichés pris en série, à faible distance les uns des autres, met en évidence l'uniformité ou la variabilité locale des peuplements. Des exemples en sont donnés.

5. Le mode de passage d'un type de peuplement à un autre peut être précisé. C'est le cas pour les limites des populations d'Ophiothrix fragilis, qui sont très nettes. L'utilisation parallèle de la télévision sous-marine apporte sur ce point un utile complément d'information.

6. Certain aspects du comportement des animaux benthiques ont été observés. Les Opbiotbrix fragilis, en population dense, prennent une attitude de capture microphage, en dressant un certain nombre de leurs bras, quand la vitesse du courant de marée ne dépasse pas 0,8 noeud en surface. Elles rabattent au contraire leurs bras quand elle est supérieure à 0,8 noeud.

\section{LITT'TÉRATURE CITÉE}

Blacker, R. W. \& Woodhead, P. M. J., 1965. A towed underwater camera. J. mar, biol. Ass. U. K. 45, 593-597.

Bolllot, G., 1960. La répartition des fonds sous-marins au large de Roscoff (Finistère). Cah. Biol. mar. 1, 3-23.

- 1964. Géologie de la Manche Occidentale. Annls Inst. océanogr., Monaco 42, 1-220.

Bouhot, G., Valerien, J., Paille, A., Boillot, G. \& Cabioch, L., 1965. Essai d'une camera légère de télévision sous-marine dans la Manche Occidentale. Res. Film 5 (4), 320-330. 
Buchanan, J. B., 1963. Mucus secretion within the spines of ophiuroids echinoderms. Proc. zool. Soc. Lond. 141, 251-260.

$\mathrm{C}_{\mathrm{ABIOCH}}$ L., 1961. Etude de la répartition des peuplements benthiques au large de Roscoff. Cab. Biol. mar. 2, 1-40.

- 1965. Quelques données sur la distribution des Hydraires et des Anthozoaires au large de Roscoff. In: Teissier, G.: Cnidaires - Cténaires. 2ième éd. (Inventaire de la faune marine de Roscoff.) Trav. Stn biol. Roscoff 16 (5), 55-57.

Craig, R. E. \& Priestley, R., 1963. Undersea photography in marine research. Mar. Res. $1963(1), 24$.

Czıнaк, G., 1959. Vorkommen und Lebensweise der Ophiothrix quinquemaculata in der nördlichen Adria bei Rovinj. Thalassia jugosl. 1 (7), 19-24.

Fontaine, A. R., 1965. The feeding mechanisms of the Ophiuroid Opbiocomina nigra. J. mar. biol. Ass. U. K. 45, 373-385.

Guille, A., 1965. Contribution à l'étude de la systématique et de l'écologie d'Ophiotbrix quinquemaculata d. Ch. Vie Milieu 15, 243-308.

MACINTYRE, A. D., 1956. The use of trawl, grab and camera in estimating marine benthos. J. mar. biol. Ass. U. K. 35, 419-429.

Nagabhushanam, A. K. \& Colman, J. S., 1959. Carrion-eating by Ophiuroids. Nature, Lond. 184 (4682), 285.

Péres, J. M. \& Picard, J. 1958. Manuel de bionomie benthique de la Mer Méditerranée. Recl Trav. Stn mar. Endoume 23 (14), 5-122.

Service Hydrographique de la Marine, 1949. Courants de marée. Manche, entrées de la Manche et de la Mer du Nord.

UexküLl, J. von, 1905. Studien über den Tonus. 2. Bewegung der Schlangensterne. Z. Biol. 46, 372-402.

Vevers, H. G., 1952. A photographic survey of certain areas of sea floor near Plymouth. J. mar. biol. Ass. U. K. 31, 215-222.

- 1956. Observations on feeding mechanisms in some echinoderms. Proc. zool. Soc. Lond. 126, 484 .

\section{Discussion suivant le rapport de CABIOCH}

ANGEL: What was the bottom current when the surface current was 0.8 knots?

CaBIOCH: Nous ne connaissons pas avec précision la vitesse du courant de fond, nous n'avons pas encore eu la possibilité d'essayer de le mesurer.

BARNES: Have you combined underwater television with underwater photography so obtaining the advantages of both techniques - a permanent record and continuous observation?

CABIOCH: Une telle expérience a été réalisée à Roscoff en 1965, nous avons pu explorer de manière continue les différents types de fonds qui ont montrés. Les résultats ont été publiés dans Research Film (BоUнот et al.).

Hohendorf: Zur Feststellung der Tauchtiefe Ihres Gerätes könnten Sie statt des Echolotes auch den Pinger verwenden. Dieser Apparat arbeitet nach einer sehr fein ansprechbaren Methode der Differenzmessung, in dem von einem Schallgeber in der Tauchtiefe des Geräts zwei Echos ausgesandt werden, eins direkt zur Oberfläche, ein zweites zum Boden. Aus der Zeitdifferenz dieser Impulse erhalten Sie stets den genauen Abstand Ihres Geräts zum Boden.

СавIOCH: Nous n'avons opéré jusqu’à présent qu'en Manche Occidentale, où les profondeurs ne dépassent pas $100 \mathrm{~m}$ et où, par conséquent, de tels dispositifs sont superflus. Il est cependant possible, de par la construction de l'appareil, d'y adjoindre un Pinger; c'est ce que nous ferons sans doute quand irons travailler à des profondeurs plus grandes.

RiEDL: Die photographischen Dokumente, die wir eben gesehen haben, geben zu besonderen Hoffnungen Anlaß. Ich möchte vermuten, daß der Ausbau dieser Geräte in zweierlei Hinsicht 
weitere und vertiefte Einsichten liefern wird. (1) Die Montage des Bildgeräts auf einem großen Schlitten mit Strecken-Zählwerk würde geschlossenen Bildserien von den Biotopgrenzen liefern. (2) Der stationäre Schlitten mit Serienbild-Schaltung und einem ins Bildfeld gebrachten Meßgerät zur Bestimmung von Stärke und Richtung der Strömung ließe die Korrelation zwischen Wasserbewegung und Schlangenstern-Verhalten quantitativ bestimmen.

Cabroch: Je retiens les suggestions intéressantes du Professeur Riedl. Il en, en effet, concevable, de modifier le bât de l'appareil et de prendre, en particulier, des vues en série en un même point. Nous n'avons pas encore eu le temps de procéder ainsi; nous n'en sommes encore qu'à l'exploration générale, préliminaire, des fonds du large de Roscoff. 